\title{
AlGaInP light-emitting diodes with omni-directionally reflecting submount
}

\author{
Thomas Gessmann, E. Fred Schubert \\ Department of Electrical, Computer, and Systems Engineering \\ Rensselaer Polytechnic Institute, Troy NY 12180 \\ John W. Graff \\ Department of Electrical and Computer Engineering \\ Boston University, Boston MA 02215 \\ and Klaus Streubel \\ Osram Opto Semiconductors Corporation \\ Regensburg, Germany
}

\begin{abstract}
:
A novel AlGaInP light-emitting diode (LED) is presented that employs high-reflectivity omnidirectional reflector (ODR) submounts. It is shown that the reflective-submount (RS) LED has a higher light-extraction efficiency than conventional LEDs. Red AlGaInP RS-LEDs bonded to Sisubstrates are demonstrated using a silver-based ODR. The ODR is perforated by an array of small-area low-resistance ohmic contacts. The optical and electrical characteristics of the RSLEDs are presented and compared to conventional AlGaInP absorbing substrates (AS) LEDs with distributed Bragg reflectors (DBR). It is shown that the light output from the RS-LED exceeds that of AS-LEDs by about a factor of two.
\end{abstract}

\section{Introduction}

AlGaInP LEDs grown on GaAs substrates are capable of emission in red to yellow-green wavelength range (Stringfellow, 1997). In order to increase the efficiency of AlGaInP LEDs, light absorption in GaAs substrates needs to be reduced or eliminated. One possibility is the insertion of an electrically conductive DBR between the LED active layers and the absorbing substrate (Stringfellow, 1997, Kato et al., 1991). However, the width of the high reflectivity stop-band of DBRs is limited; moreover, at oblique incidence angles of incidence, DBRs become transparent resulting in optical losses. To improve the DBR properties, several technical approaches have been proposed and demonstrated including the use of disordered DBRs (Popov et al., 1997; Chiou et al., 2000; Xu et al., 2001)

Another possibility is to replace the absorbing substrate (AS) by a transparent substrate (TS), preferably GaP (Krames et al., 1999; Kish et al. 1994). To date, TS devices with external efficiencies as high as $55 \%$ have been reported (Krames et al., 1999). TS-LEDs exceed the performance of AS-LEDs by approximately a factor of two (Kish et al. 1994). However, the TS approach suffers from the use of costly $\mathrm{GaP}$ substrates and a complicated wafer bonding process (Kish et al., 1995). Yet another way of improving AlGaInP device performance is the use of 
wafer-fused thin film LEDs with metal micro-reflectors for significantly increased light extraction (Streubel et al., 2002). The thin-film LEDs discussed by Streubel et al. (2002) incorporate micro-reflectors that redirect waveguided modes towards the chip surface.

\section{Description of reflective-submount (RS) AlGaInP LED technology}

Here we report on a very promising new type of AlGaInP LED. The device employs a highreflectivity, planar, omni-directional reflector (ODR). This allows for light emitted towards the substrate at any angle of incidence at the substrate-epilayer interface to be reflected at the substrate-epilayer interface towards the top surface of the chip. It is shown that this type of LED has the potential of outperforming AS-LED and possibly TS-LED devices. In addition, the RSLED technology offers cost advantages over TS-LED technology.

The approach employs a new processing scheme in which the AlGaInP wafer is combined with an ODR, and bonded to a Si wafer. Other submounts such as a metal substrate would also be possible. The expected advantages of RS-LED technology are:

- No absorption in the substrate due to presence of high-reflectivity ODR

- High light extraction and luminous efficiency

- Low current density under top contact due to patterned back-side contact and absence of current-spreading substrate

- Suitable for high-power operation due to high thermal conductivity of $\mathrm{Si}$ or metal submount

- Low-cost manufacturing due to employment of low-cost Si wafer or metal submounts

- High yield due to simplicity of wafer bonding process

One of the key innovations of the RS-LED is a high-reflectivity omni-directional reflector $(O D R)$. This reflector may be realized with the combination of a low-refractive-index dielectric layer and a metal layer, or by the use of a high reflectivity metal layer alone. It is shown that this reflector has very high reflectivity for all angles of incidence, $i$. $e$., is omni-directional. In comparison, a DBR mirror has only a limited range of angles for which high reflectivity is achieved.

The schematic structure of the RS-LED is shown in Fig. 1. It consists of a top currentspreading layer (or window layer), the active layers, a bottom window layer, the ODR, and a low-cost Si wafer used as a conductive submount. The active layers include the lower and upper confinement layers and the bulk or multiple-quantum well (MQW) active region. The wafer is grown in the standard "p-side up" mode that is employed in nearly all state-of-the-art AlGaInP LEDs. Therefore the epi-growth of RS-LEDs uses well-established epitaxial technology.

As shown in Fig. 1, the ODR is penetrated by many small ohmic contacts that cover only a small fraction of the backside area of the LED die. Assuming that the ohmic contacts have an area of $1 \%$ of the die, and that the alloyed ohmic contact metal is $50 \%$ reflective, a reduction in reflectivity of only $0.5 \%$ is obtained due to the ohmic contacts. As will be shown later, a reflectance of $99 \%$ can be obtained with the structure presented here. 
Typical contact resistances for n-type ohmic contacts to arsenide and phosphide based semiconductors range between $10^{-6}$ and $10^{-8} \Omega \mathrm{cm}^{2}$. Assuming an LED die area of $250 \mu \mathrm{m} \times$ $250 \mu \mathrm{m}$ and ohmic contact coverage of $1 \%$ (total area of $25 \mu \mathrm{m} \times 25 \mu \mathrm{m}$ ), we calculate a contact resistance of $R_{\mathrm{c}}=\rho_{\mathrm{c}} / A$ (where $A$ is the contact area) of $1.6 \mathrm{~m} \Omega$ to $0.16 \Omega$. Assuming a drive current of $25 \mathrm{~mA}$, the voltage drop across the n-type contact amounts to only $4 \mathrm{mV}$ in the worst case (i. e. for $\rho_{\mathrm{c}}=10^{-6} \Omega \mathrm{cm}^{2}$ ). Even if the LED were driven at $100 \mathrm{~mA}$, the voltage drop across the contact resistance would be $16 \mathrm{mV}$, still very small. For the lowest assumed specific contact resistance, i.e. $10^{-8} \Omega \mathrm{cm}^{2}$, the voltage drop at a drive current of $100 \mathrm{~mA}$ would be $0.16 \mathrm{mV}$, again a negligibly small value.
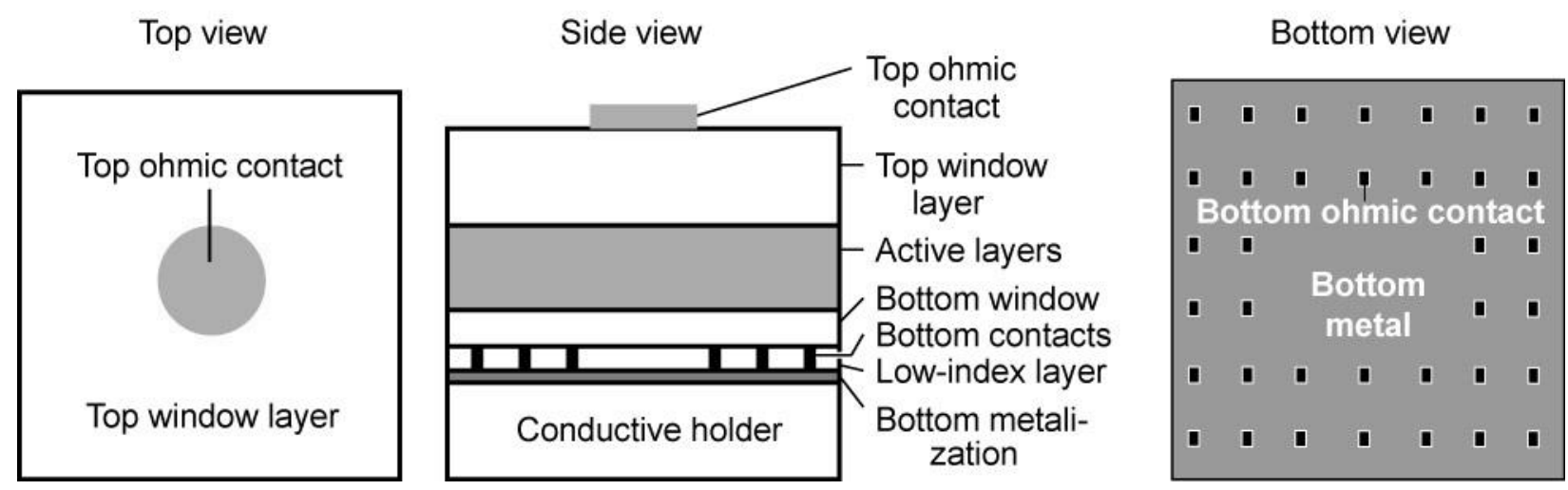

Fig. 1. Top view, side view, and bottom view of the RS (reflective submount) LED structure. The structure comprises a top ohmic contact, a top window layer, the active layers (a top cladding layer, an active light-emitting region, and a bottom cladding layer), a bottom window layer, a transparent low-index layer (for example silicon dioxide) penetrated by ohmic contacts, a bottom metalization layer, and a conductive holder.

\section{Fabrication process for RS-LEDs}

There are several processes that can be used to fabricate RS-LEDs. One of these processes is shown schematically in Figs. 2. The process uses "p-side up" epiwafers, which are the standard for epitaxial growth. The process includes deposition of the ODR, bonding of the wafer to a submount (Si or metal wafer), removal of the GaAs substrate, and front-side processing, followed by dicing and packaging. Note that the current flow under the top contact is minimized due to the contact geometry of the backside contact. 
(a) Growth

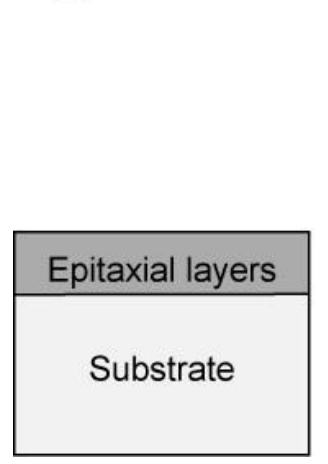

(b) Back-side processing

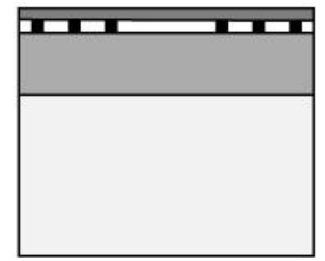

(c) Bonding to holder

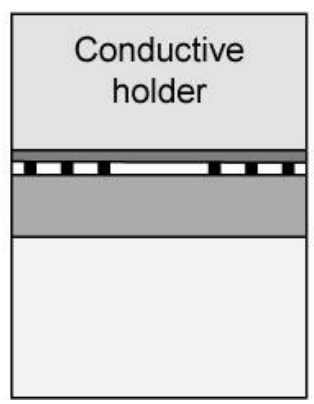

(d) Substrate removal

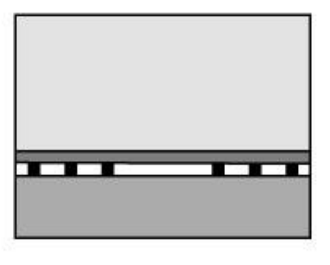

(e) Front-side processing

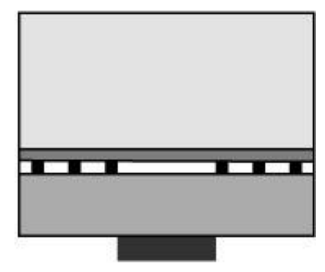

Fig. 2. Processing sequence of the reflective submount (RS) light-emitting diode using "p-side-up" epitaxial growth. After epitaxial growth (a), the back-side of the wafer is processed (b). The wafer is then bonded to the conductive holder (c) followed by substrate removal (d) and front-side processing (e). Note that the top p-side of the epilayers becomes the backside of the LED.

In another possible process, the ODR is deposited on the n-type side of the active layers after the $\mathrm{p}$-side of the wafer has been mounted on a temporary holder and the substrate has been removed. Each process has advantages and disadvantages. The process employing a temporary holder involves more steps, but has the potential for lower contact resistances for the backside contact (ODR side contact) of the LED. This is because the backside contact is an n-type contact. The process shown in Fig. 2 does not require a temporary holder but the backside ohmic contacts are to p-type material, which may result in higher contact resistances.

Of course, an "n-side up" growth process can also be envisioned. However, this would require the development of entirely new epitaxial growth processes.

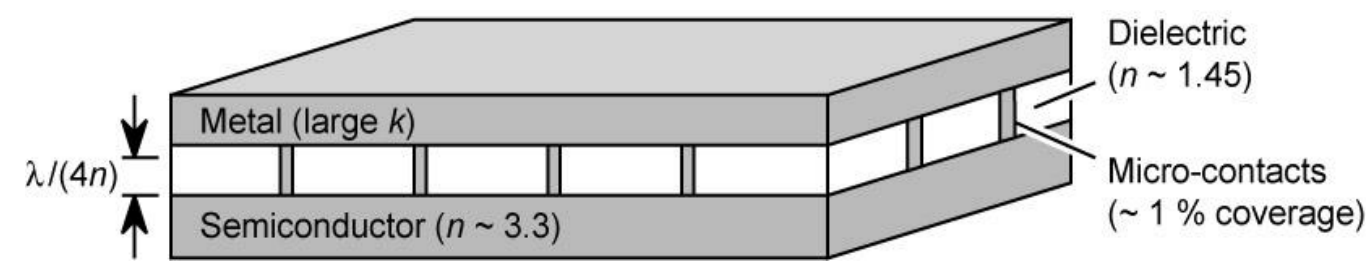

Fig. 3. Structure of high-reflectivity omni-directional reflector composed of a semiconductor, a low-refractive-index layer perforated by an array of electrical microcontacts, and a reflective metal layer.

\section{RS-LED omni-directional reflector technology}

Recently, several concepts for high-reflectivity ODRs were presented and demonstrated (Fink et al., 1998; Weber et al., 2000; Deopura et al., 2001). Dielectric and polymer ODRs have much lower losses than metal ODRs (Fink et al., 1998). However a hybrid ODR, as used here, can 
provide low loss as well. The high-reflectivity omni-directional reflector considered here is composed of the semiconductor, a low-refractive-index layer, and a reflective metal layer, as shown in Fig. 3. The normal-incidence reflectance of the triple-layer omni-directional reflector is given by (Ma et al., 2001)

$$
R=\frac{\left\{\left(n_{\mathrm{S}}-n_{\mathrm{li}}\right)\left(n_{\mathrm{li}}+n_{\mathrm{m}}\right)+\left(n_{\mathrm{S}}+n_{\mathrm{li}}\right) k_{\mathrm{m}}\right\}^{2}+\left\{\left(n_{\mathrm{S}}-n_{\mathrm{li}}\right) k_{\mathrm{m}}+\left(n_{\mathrm{S}}+n_{\mathrm{li}}\right)\left(n_{\mathrm{li}}-n_{\mathrm{m}}\right)\right\}^{2}}{\left\{\left(n_{\mathrm{S}}+n_{\mathrm{li}}\right)\left(n_{\mathrm{li}}+n_{\mathrm{m}}\right)+\left(n_{\mathrm{S}}-n_{\mathrm{li}}\right) k_{\mathrm{m}}\right\}^{2}+\left\{\left(n_{\mathrm{S}}+n_{\mathrm{li}}\right) k_{\mathrm{m}}+\left(n_{\mathrm{S}}-n_{\mathrm{li}}\right)\left(n_{\mathrm{li}}-n_{\mathrm{m}}\right)\right\}^{2}}
$$

where $n_{\mathrm{s}}, n_{\mathrm{li}}$, and $n_{\mathrm{m}}$ are the refractive indices of the semiconductor, low-index layer, and metal, respectively; $k_{\mathrm{m}}$ denotes the extinction coefficient of the metal. Eq. (1) is calculated for a thickness $d=\lambda /\left(4 n_{\mathrm{li}}\right)$ of the low-index layer ("quarterwave layer") with $\lambda$ being the wavelength of the incident light in vacuum. Evaluation of Eq. (1) yields a normal-incidence reflectance of $>99 \%$ for an AlGaInP / $\mathrm{SiO}_{2} / \mathrm{Ag}$ structure. The low-index layer is penetrated by small-area alloyed ohmic contacts that cover an area of $1 \%$ of the backside of the wafer. Assuming that the ohmic contacts have a low reflectivity, the ODR reflectivity is reduced by about $1 \%$.

The semiconductors used for DBRs in AS-LEDs have Brewster angles that are much less than $90^{\circ}$. As a result, light emitted near the Brewster angle from the active region towards the substrate is not reflected but transmitted through the DBR and absorbed in the GaAs substrate. Furthermore, optical modes guided in the semiconductor epitaxial layer do not propagate for large distances due to the low reflectance of the DBR. Light absorption in the substrate and loss of waveguiding modes lead to a significant loss in light extraction efficiency.

High-reflectivity ODRs can be designed for a variety of incident media in such a way that the Brewster angle $\theta_{\mathrm{B}}$ becomes $90^{\circ}$ or even becomes imaginary. In both cases the above-mentioned loss mechanisms are avoided. The ODR reflects all light emitted from the active region towards the substrate back to the top of the structure. Furthermore, waveguided modes caused by total internal reflection at the semiconductor-air interface have no substantial loss when using a highreflectivity ODR. This is particularly important for devices that are scaled to large chip sizes. Presently, large-area devices are known to suffer from scaling-induced loss in efficiency.

Omni-directional reflectivity can be attained in DBR structures employing birefringent transparent polymers (Weber et al., 2000) or high-index-contrast materials with a very wide high-reflection band (Fink et al., 1998).

A system consisting of a metal layer covered by a semiconductor can be an ODR as well since the Brewster angle

$$
\tan \theta_{\mathrm{B}}=\frac{n_{\mathrm{m}}-i k_{\mathrm{m}}}{n_{\mathrm{s}}}
$$

has an imaginary part which is usually much larger than its real part. In spite of absorption in the metal, high reflectivity can be maintained if the metal refractive index $n_{\mathrm{m}}$ is sufficiently different from $n_{\mathrm{s}}$. From Eq. (2) follows that the condition $n_{\mathrm{m}}<n_{\mathrm{S}}$ is preferable. A strong increase in 
reflectivity can be attained by inserting a transparent quarter-wave layer with small refractive index between metal and semiconductor.
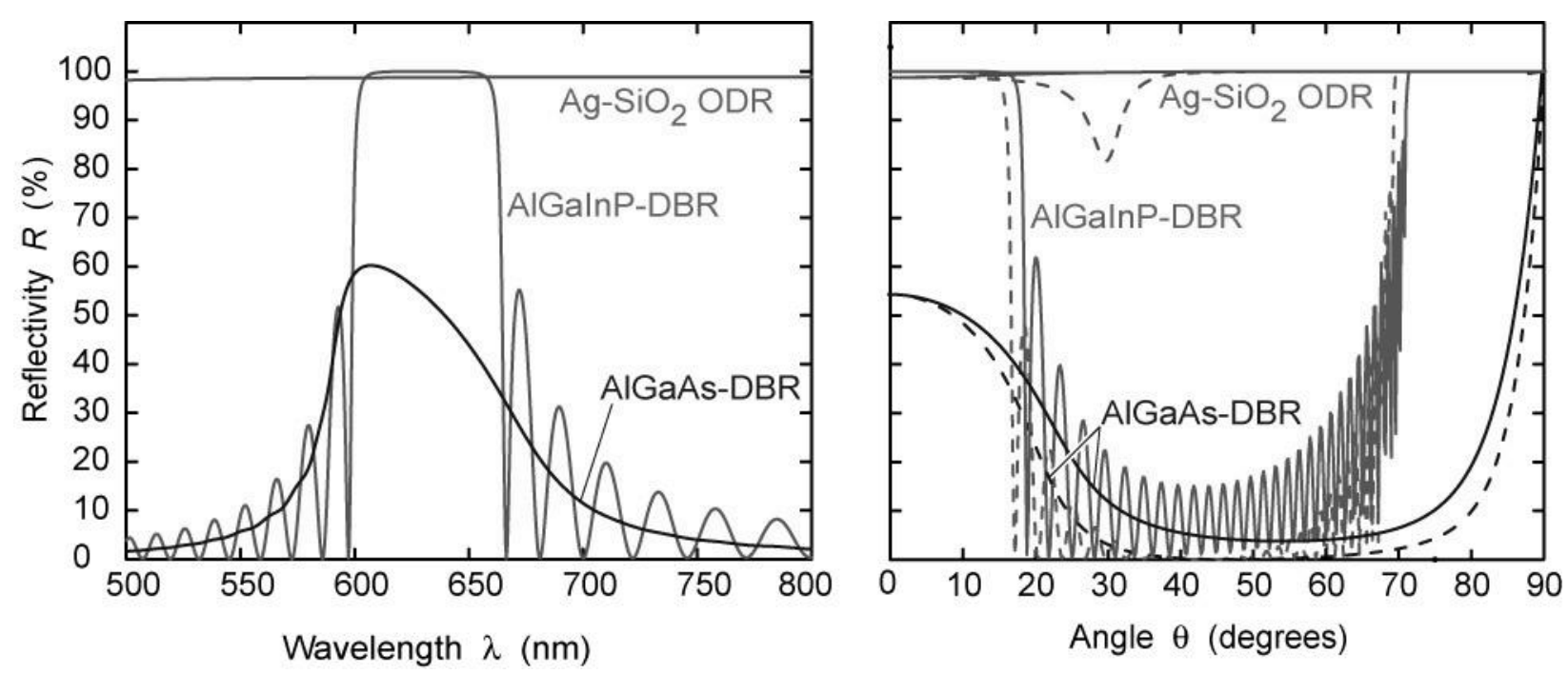

Fig. 4. Calculated reflectivity for an Ag-ODR and two different DBRs consisting of quarter-wave stacks with a Bragg wavelength at $630 \mathrm{~nm}$. GaP was chosen as the external medium. The absorbing AlGaAs-DBR is composed of $35\left(\mathrm{Al}_{0.91} \mathrm{Ga}_{0.09} \mathrm{As} / \mathrm{Al}_{0.09} \mathrm{Ga}_{0.91} \mathrm{As}\right)$ pairs, the transparent AlGaInP-DBR consists of $35\left[\left(\mathrm{Al}_{0.3} \mathrm{Ga}_{0.7}\right)_{0.5} \mathrm{In}_{0.5} \mathrm{P} / \mathrm{Al}_{0.5} \mathrm{In}_{0.5} \mathrm{P}\right]$ pairs. The Ag-ODR has a $500 \mathrm{~nm}$ thick Ag-layer covered by a quarterwave-thick $\mathrm{SiO}_{2}$ layer on a GaAs substrate. (a) shows the reflectivity versus wavelength for normal incidence. (b) depicts the angular dependence of the reflectivity at $630 \mathrm{~nm}$; the solid and dashed lines correspond to $\mathrm{s}$ - and $\mathrm{p}$-polarized waves, respectively

The angle-dependence of the reflectance of the ODR is very small. High-reflectivity DBRs and an ODR are compared in Fig. 4 as a function of wavelength (Fig. 4a) as well as angle of incidence (Fig. 4b). Comparison of the theoretical spectra reveals ODR characteristics that are significantly better than those of the DBR. This applies in particular to incidence angles in the region around $45^{\circ}$ where the ODR can be more than an order of magnitude better than conventional DBRs.

A figure of merit for ODRs or DBRs employed in an LED structure is the reflectivity integrated over all incident angles $\theta$

$$
R_{\text {int }}(\lambda)=\frac{1}{2 \pi} \int_{0}^{\pi / 2} R(\lambda, \theta) 2 \pi \sin \theta \mathrm{d} \theta
$$

Values of $R_{\text {int }}$ at $\lambda=630 \mathrm{~nm}$ are summarized in Table 1 for different types of high-reflectivity metal-ODRs and for the DBRs shown in Fig. 4. Inspection of Table 1 shows that the best ODR 
based on a semiconductor $/ \mathrm{SiO}_{2} / \mathrm{Ag}$ structure is by at least a factor of two better than the DBRs.

\begin{tabular}{|c|c|c|c|}
\hline Type of reflector & $\begin{array}{c}\text { Refractive index } \\
(\lambda=630 \mathrm{~nm})\end{array}$ & $\begin{array}{c}\text { Extinction coefficient } \\
(\lambda=630 \mathrm{~nm})\end{array}$ & $\begin{array}{c}R_{\mathrm{int}} \\
(\lambda=630 \mathrm{~nm})\end{array}$ \\
\hline $\mathrm{Ag}-\mathrm{SiO} 2 \mathrm{ODR}$ & $n_{\mathrm{m}}=0.08$ & $k_{\mathrm{m}}=4$ & 0.994 \\
\hline $\mathrm{Au}-\mathrm{SiO} 2 \mathrm{ODR}$ & $n_{\mathrm{m}}=0.19$ & $k_{\mathrm{m}}=3$ & 0.971 \\
\hline $\mathrm{Al}-\mathrm{SiO} 2 \mathrm{ODR}$ & $n_{\mathrm{m}}=1.38$ & $k_{\mathrm{m}}=7.7$ & 0.983 \\
\hline $\mathrm{AlGaAs}-\mathrm{DBR}$ & $n_{1}=3.1, n_{\mathrm{h}}=3.8$ & $k_{\mathrm{h}}=0.15$ & 0.14 \\
\hline $\mathrm{AlGaInP}-\mathrm{DBR}$ & $n_{1}=2.9, n_{\mathrm{h}}=3.35$ & transparent & 0.473 \\
\hline
\end{tabular}

Table 1. Values of the angle-integrated reflectivity $R_{\text {int }}$ at $630 \mathrm{~nm}$ calculated from Eq. (3) using theory curves such as shown in Fig. 4b. The metal-ODRs consist of a $500 \mathrm{~nm}$ thick metal layer covered by a quarterwave $\mathrm{SiO}_{2}$ layer on a $\mathrm{GaAs}$ substrate. The absorbing AlGaAs-DBR is composed of $35\left(\mathrm{Al}_{0.91} \mathrm{Ga}_{0.09} \mathrm{As} / \mathrm{Al}_{0.09} \mathrm{Ga}_{0.91} \mathrm{As}\right)$ pairs, the transparent AlGaInP-DBR consists of $35\left[\left(\mathrm{Al}_{0.3} \mathrm{Ga}_{0.7}\right)_{0.5} \mathrm{In}_{0.5} \mathrm{P} / \mathrm{Al}_{0.5} \mathrm{In}_{0.5} \mathrm{P}\right]$ pairs. The values of $n_{\mathrm{m}}, n_{\mathrm{l}}$, $n_{\mathrm{h}}$ correspond to the refractive indices of the metals and the low-/high-index layers in the DBRs, $k_{\mathrm{m}}$ and $k_{\mathrm{h}}$ denote the metal- and DBR-extinction coefficients. GaP $(n=3.0)$ was chosen as outside medium.
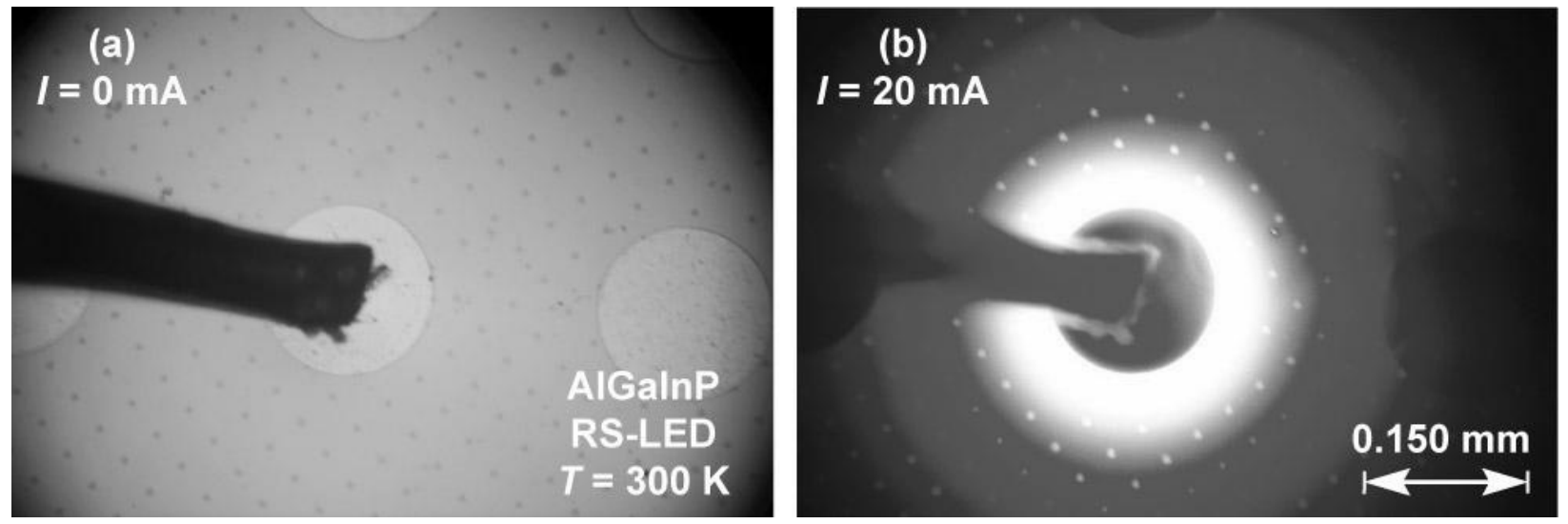

Fig. 5. (a) Micrograph of AlGaInP RS-LED without applied voltage. (b) Micrograph of RS-LED under current injection $(I=20 \mathrm{~mA})$.

\section{Experimental results on RS-LEDs and comparison with other LEDs}

A micrograph of an RS-LED is shown in Fig. 5 without applied voltage (Fig. 5a) and under operation (Fig. 5b). The figure reveals that the emission occurs in the red part of the optical spectrum. The pattern of small area contacts beneath the large area top contacts can be clearly seen. In Fig. 5 (a), the small-area contacts appear dark because their reflectivity is significantly 
smaller than the reflectivity of the surrounding silver metal. In Fig. 5 (b), however, the small contacts are brighter than the surrounding area indicating current crowding in the vicinity of the small contacts. The top contact has a diameter of $150 \mu \mathrm{m}$.

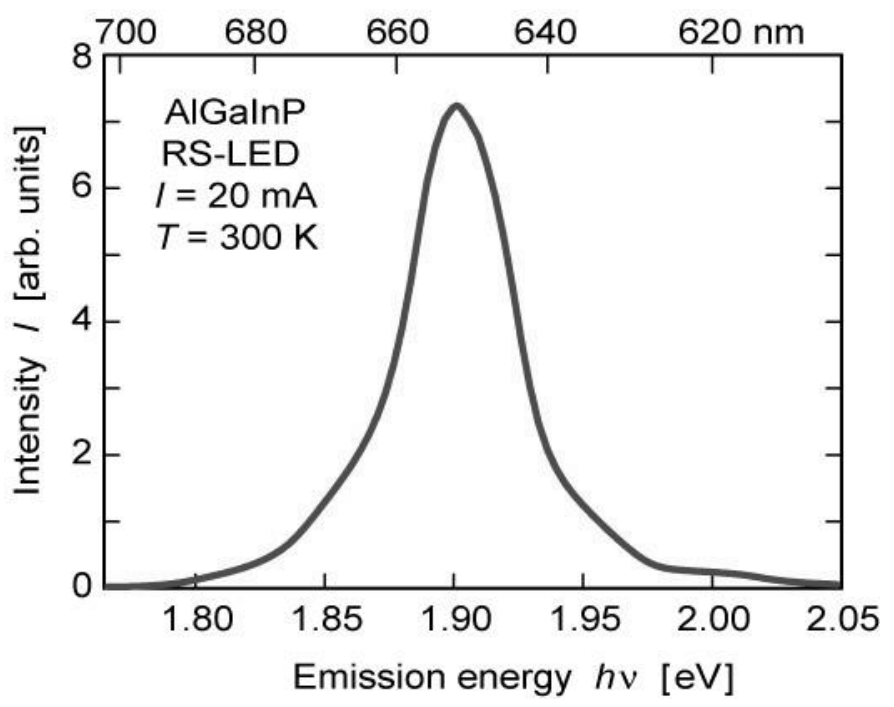

Fig. 6. Room temperature emission spectrum of an AlGaInP RS-LED at an injection current of $20 \mathrm{~mA}$.

The room temperature emission spectrum of the AlGaInP RS-LED is shown in Fig. 6. The device emits in the red with a peak emission wavelength of $652 \mathrm{~nm}$. The full-width at halfmaximum of the emission band is about $15 \mathrm{~nm}$.

Commercial AlGaInP AS-LEDs emitting at 630 to $650 \mathrm{~nm}$ were used as a reference to evaluate the optical properties of the RS-LED. The AS-LEDs were grown either directly on an absorbing n-type GaAs substrate or on a conductive DBR covering the substrate. These ASLEDs devices had a circular top contact $(150 \mu \mathrm{m}$ diameter) and the backside contact was a $\mathrm{AuGe} / \mathrm{Ni} / \mathrm{Au}$ layer deposited by e-beam evaporation on the n-type GaAs substrate and annealed at $450{ }^{\circ} \mathrm{C}$ for $30 \mathrm{sec}$ in forming gas.

Measurements of the optical output power versus injection current of an RS-LED and an ASLEDs with a DBR are shown in Fig. 7. As can be seen in the figure, the output power of the RSLED is significantly larger than that of the DBR-enhanced AS-LEDs. The AS-LED without the DBR has a lower output power than the AS-LED with DBR and is therefore not shown in the figure.

The maximum light output extracted from the RS-LED is much larger than the light output of the AS-LEDs. We attribute the higher power of RS-LEDs to the lower power loss of waveguided light rays. The optical losses are much less in a device employing an omni-directional reflector instead of a DBR. Therefore the attenuation of waveguided light traveling towards the 
edges of an LED die is much smaller in the case of a RS-LED. This effect can result a significantly enhanced light output since most light generated in the active region is emitted into waveguided modes. Note that the light output from the DBR-enhanced AS-LED exceeds that of the standard LED (not shown in the figure) by more than a factor of two.

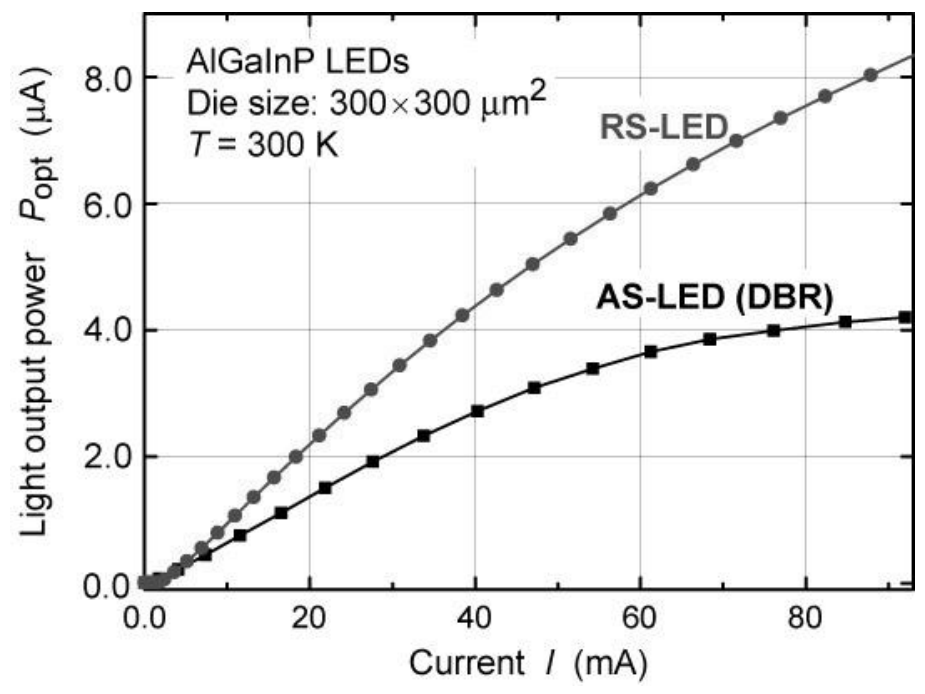

Fig. 7. Dependence of the optical output power $L$ on the drive current $I$ for RS- and ASLEDs. The output power $L$ is given as the photodetector current measured with the sample placed inside of an integrating sphere.

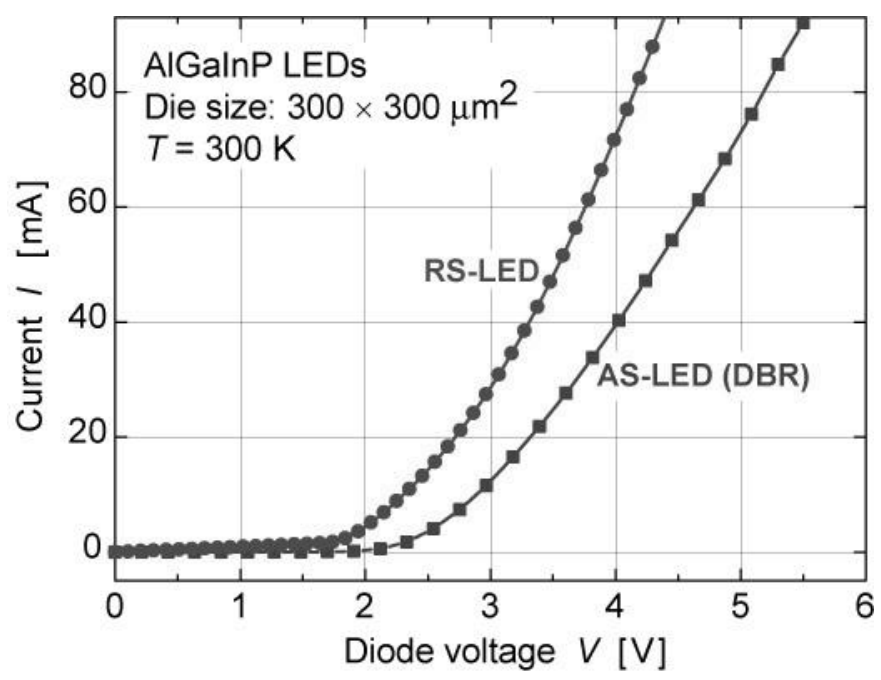

Fig. 8. Current-voltage characteristic of an AlGaInP RS-LED and AS-LEDs with and without a distributed Bragg reflector (DBR). 
The current-voltage (I-V) characteristic of an AlGaInP RS-LED is shown in Fig. 8. At a current of $20 \mathrm{~mA}$, the forward voltage is about $2.7 \mathrm{~V}$. For comparison, the I-V characteristic of an AlGaInP AS-LED with a DBR is also shown in the figure. It is important to note that the I-V's shown in the figure do not result from optimized processes or optimized top layers for low ohmic contact resistances. However, the figure clearly illustrates that, for the current LED fabrication process, the RS-LED does not have a forward-voltage penalty. It is expected that the forward voltage of RS-LEDs will decrease to values near or, more likely, below $2.0 \mathrm{~V}$ for an optimized fabrication process.

Electroluminescence spectra of a RS-LED and a TS-LED emitting at $590 \mathrm{~nm}$ are shown in Fig. 9. The spectra were measured at two different drive current values in order to study the effect of active region heating on both devices. Devices with a size of $(300 \times 300) \mu \mathrm{m}^{2}$ were isolated by wet-chemical etching (RS-LED) or cleaved from the wafer (TS-LED). The slope of the high-energy side of the electroluminescence band is proportional to

$$
\text { Intensity } \propto \exp -(E / k T)
$$

where $k$ is Boltzmann's constant, and $T$ is the temperature. As the straight-line fits in Fig. 9 show the slope indeed becomes smaller with increasing value of the drive current indicating a temperature rise with injection current. This effect is more pronounced for the TS-LED indicating stronger heating of the device.

We attribute the lower device heating of the RS-LED to the higher thermal conductivity of the $\mathrm{Si}$ substrate. The thermal conductivity of $\mathrm{Si}$ is about $300 \%$ larger than in GaAs and about $50 \%$ higher than in GaP. As a result, the active region is less heated in RS-LEDs as compared to AS- and TS-LEDs. Based on the smaller temperature increase in RS-devices and the higher thermal conductivity of $\mathrm{Si}$ as compared to GaAs and GaP, we expect RS-devices can be operated at higher injection currents and higher optical power levels. This is an especially important characteristic for future high-power LEDs. 

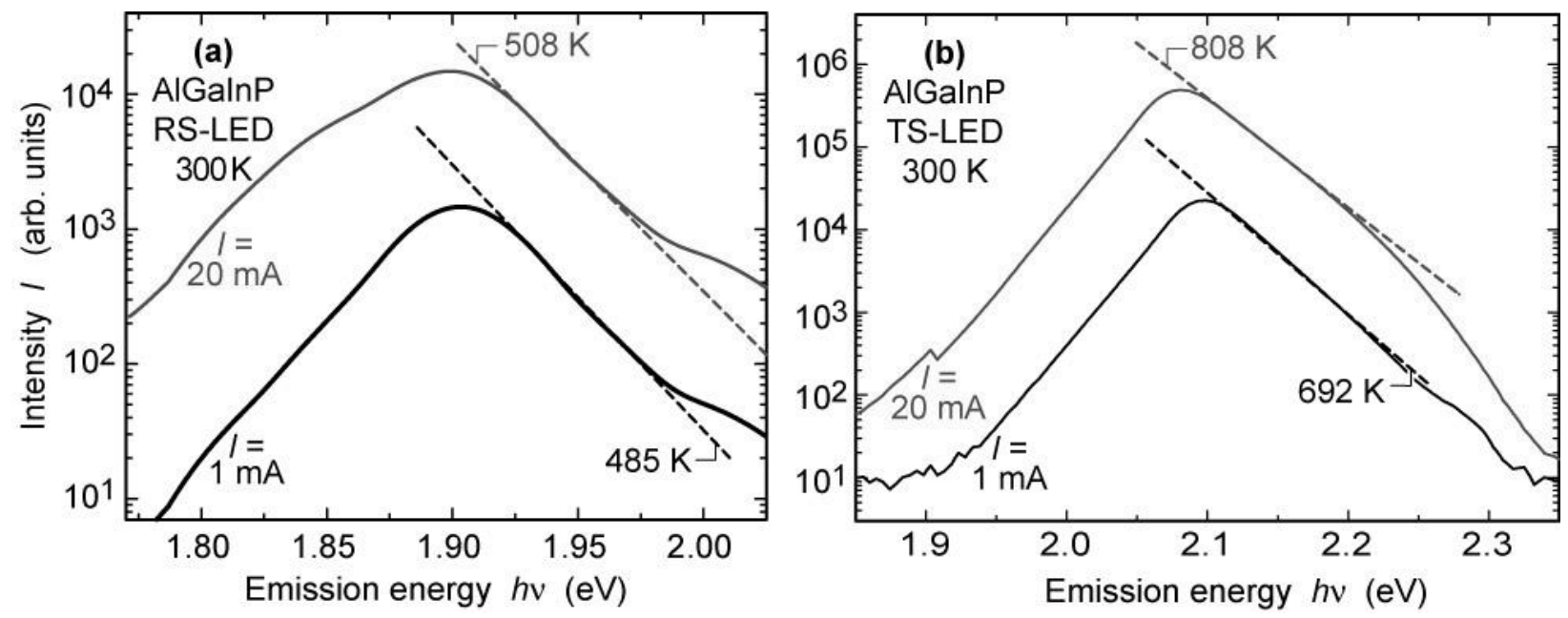

Fig. 9. (a) Electroluminescence spectra of a RS-LED emitting at $650 \mathrm{~nm}$ with a device size of $(300 \times 300) \mu \mathrm{m}^{2}$. The straight lines represent fits to the high-energy side of the spectrum. (b) Electroluminescence spectra of a TS-LED emitting at $590 \mathrm{~nm}$ with a device size of $(300 \times 300) \mu \mathrm{m}^{2}$. The straight lines represent fits to the high-energy side of the spectrum.

\section{Comparison of RS-LEDs to AS-, TS-LED, and other AlGaInP LED technologies}

Optical losses in AS-LED technology can be large even if a DBR is employed. The RS-LED technology does not exhibit these optical losses due to the employment of a high-reflectivity omni-directional reflector. Therefore we anticipate a significant performance advantage for RSLED versus AS-LED technology.

(a) Absorbing substrate (AS)

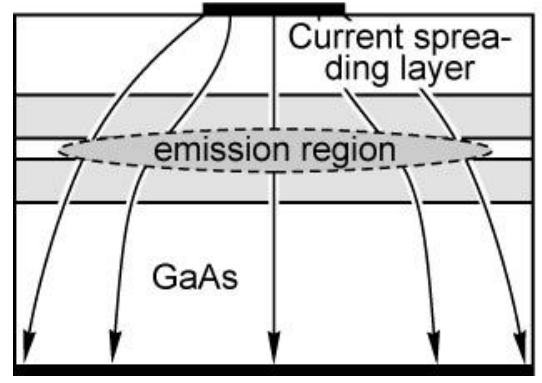

(b) Transparent substrate (TS)

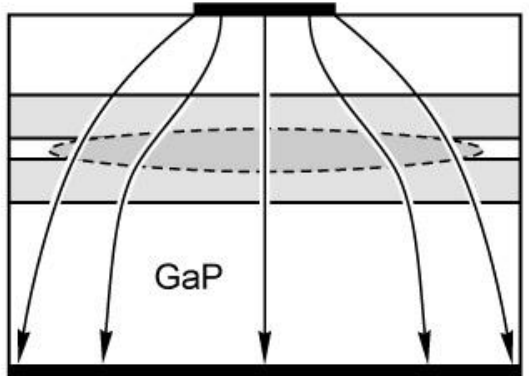

(c) Reflective submount (RS)

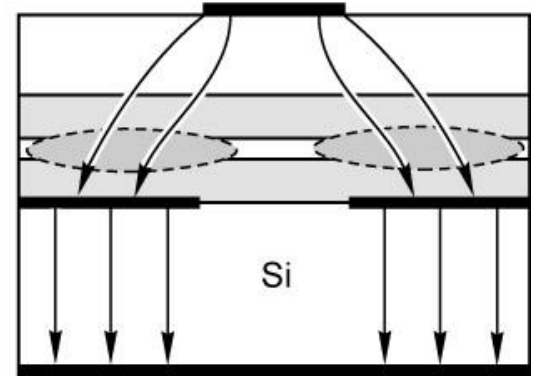

Fig. 10. Schematic illustration of current distribution in AS, TS, and RS technology LEDs. Note that in the AS and TS-LEDs, the current density is maximal under the top contact. 
In addition, the RS-LED technology could become superior to TS-LED technology. Specifically, the RS-LED technology is expected to display lower fabrication cost and potentially higher luminous performance. The reasons for these expectations are as follows:

- RS-LEDs have limited current injection under the top contact. Figure 10 compares the current injection patterns for AS, TS, and RS technologies. Inspection of the figure reveals that both AS and TS technologies have maximum current density under the top contact. Since the top contact is of low reflectivity, light generated under it may be blocked by the contact itself. The RS-LED avoids these optical losses due to a patterned backside contact that results in a current injection pattern minimizing the current flow under the top contact. Note that a backside contact patterning would not be beneficial to AS and TS-LEDs because the thick and highly conductive substrates ( $\mathrm{GaAs}$ and $\mathrm{GaP}$ ) very effectively spread the current along lateral directions.

- High thermal conductivity of Si submounts. The Si submount employed in the RS-LED has a higher thermal conductivity than either GaAs or GaP substrates. Table 2 compares the thermal conductivity of these three materials.

\begin{tabular}{|c|c|c|c|}
\hline Material & GaAs & GaP & Si \\
\hline Thermal conductivity & $0.5 \mathrm{~W} /(\mathrm{cm} \mathrm{K})$ & $1.1 \mathrm{~W} /(\mathrm{cm} \mathrm{K})$ & $1.5 \mathrm{~W} /(\mathrm{cm} \mathrm{K})$ \\
\hline
\end{tabular}

Table 2. Thermal conductivity of GaAs, GaP, and $\mathrm{Si}$

The higher thermal conductivity of $\mathrm{Si}$ submounts will allow for lower active region temperatures and thus potentially higher drive currents. Furthermore, the greater mechanical stability of Si wafers will enable thinner submounts as compared to TS or AS devices, thus allowing for improved heat sinking. Calculations (Gessmann et al., 2001) have shown that the low-index dielectric layer to be used for the ODR has negligible influence on the thermal conductance due to its small thickness $(\lambda / 4 n \approx 100 \mathrm{~nm})$.

- Lower cost of Si substrates. Si or metal substrates have a significant cost advantage over $\mathrm{GaP}$ substrates used in TS-LED technology.

- High-yield bonding process. The RS wafer bonding process is likely to have excellent yield. It is well known that the TS GaP wafer-bonding process is complicated and has a number of crucial requirements. In contrast, the RS wafer bonding process is simple, mainly because it is a metal-to-metal and not a semiconductor-to-semiconductor bonding process.

Surface texturing of LEDs is a well-known technique that allows for higher extraction efficiency. Such surface texturing could also be applied to RS-LEDs for further enhancement of the output power. 
Acknowledgment: This work was supported in part by the National Science Foundation (Dr. Varshney), the US Army Research Office/Defense Advanced Projects Agency (Dr. Carrano), the University of New Mexico/Office of Naval Research (Drs. Wood and Hersee).

\section{References}

Chang S. J. and Chang C. S. "AlGaInP-GaInP compressively strained multiquantum well lightemitting diodes for polymer fiber applications" IEEE Photonics Technol. Lett. 10, 772 (1998a)

Chang S. J. and Chang C. S. "650 nm AlGaInP / GaInP compressively strained multi-quantum well light emitting diodes" Jpn. J. Appl. Phys 37, L653 (1998b)

Chen C. H., Stockman S. A., Peanasky M. J., and Kuo C. P. "OMVPE growth of AlGaInP for high-efficiency visible light-emitting diodes" in Semiconductor and Semimetals 48, 97 (1997)

Chiou S. W., Lee C. P., Huang C. K., and Chen C. W. "Wide-angle distributed Bragg reflectors for $590 \mathrm{~nm}$ amber AlGaInP light-emitting diodes" J. Appl. Phys. 87, 2052 (2000)

Deopura M., Ullal C. K., Temelkuran B., and Fink Y. "Dielectric omni-directional visible reflector" Optics Letters 26, 1197 (2001)

Fink Y., Winn J. N., Shanhui F., Chiping Ch., Michel J., Joannopoulos J. D., Thomas E. L. "A dielectric omni-directional mirror" Science 282, 1679 (1998)

Gessmann T., Graff J. W., and Schubert E. F., unpublished results (2001)

Jones, E. D., "Light emitting diodes for general illumination," LED Solid State Lighting Workshop Report, Washington, D.C., Optoelectronics Industry Development Association (OIDA), March 2001.

Kato T., Susawa H., Hirotani M., Saka T., Ohashi Y., Shichi E., and Shibata S. "GaAs/AlGaAs surface emitting IR LED with Bragg reflector grown by MOCVD” J. Cryst. Growth 107, 832 (1991)

Kish F. A., Steranka F. M., DeFevere D. C., Vanderwater D. A., Park K. G., Kuo C. P., Osentowski T. D., Peanasky M. J., Yu J. G., Fletcher R. M., Steigerwald D. A., Craford M. G., and Robbins V. M. "Very high-efficiency semiconductor wafer-bonded transparentsubstrate $\left(\mathrm{Al}_{x} \mathrm{Ga}_{1-x}\right)_{0.5} \mathrm{In}_{0.5} \mathrm{P} / \mathrm{GaP}$ light-emitting diodes" Appl. Phys. Lett. 64, 2839 (1994)

Kish F. A., Vanderwater D. A., Peanasky M. J., Ludowise M. J., Hummel S. G., and Rosner S. J. "Low-resistance Ohmic conduction across compound semiconductor wafer-bonded interfaces" Appl. Phys. Lett. 67, 2060 (1995)

Kish F. A. and Fletcher R. M. "AlGaInP light emitting diodes" in "High Brightness Light Emitting Diodes" Semiconductor and Semimetals Vol. 48, p 149, Eds. G. B. Stringfellow and M. G. Craford (Academic Press, San Diego, 1997)

Krames M. R. et al. "High-power truncated-inverted-pyramid $\left(\mathrm{Al}_{x} \mathrm{Ga}_{1-x}\right)_{0.5} \operatorname{In}_{0.5} \mathrm{P} / \mathrm{GaP}$ lightemitting diodes exhibiting > $50 \%$ external quantum efficiency” Appl. Phys. Lett. 75, 2365 (1999)

Krames M. R., Amano H., Brown J. J., and Heremans P. L. "High-efficiency light-emitting diodes" IEEE J. Select. Topics Quant. Electronics 8, 185 (2002) 
Kuo C. P., Fletcher R. M., Osentowski T. D., Lardizabel M. C., Craford M. G., and Robbins V. M. "High performance AlGaInP visible light-emitting diodes" Appl. Phys. Lett. 57, 2937 (1990)

Ma Z., Gessmann T., Graff J. W., and Schubert E. F. unpublished results (2001)

Mueller G. "Electroluminescence I" Semiconductor and Semimetals Vol. 64 (Academic Press, San Diego, 2000)

Popov K. V., Dobrowolski J. A., Tikhonravov A. V., and Sullivan B. T. "Broadband highreflection multilayer coatings at oblique angles of incidence" Applied Optics 36, 2139 (1997)

Schubert E. F. "Light Emitting Diodes," (Cambridge University Press, Cambridge, 2003)

Shah J., Graff J. W., and Gessmann T., unpublished results (2001)

Streubel K., Linder N., Wirth R., and Jaeger A. "High brightness AlGaInP light-emitting diodes" IEEE J. Select. Topics Quant. Electronics 8, 321 (2002)

Stringfellow G. B. and Craford M. G. "High brightness light emitting diodes" Semiconductor and Semimetals Vol. 48 (Academic Press, San Diego, 1997)

Weber M. F., Stover C. A., Gilbert L.A., Nevitt T. J., Ouderkirk A. J. "Giant birefringent optics in multilayer polymer mirrors" Science 287, 2451 (2000)

$\mathrm{Xu}$ J., Fang H., and Lin Zh. "Expanding high-reflection range in a dielectric multilayer reflector by disorder and inhomogeneity" J. Phys D: Appl. Phys. 34, 445 (2001) 